\title{
On the inversion of sound channel data
}

\author{
M. R. Osborne* \\ (Received 7 August 2000)
}

\begin{abstract}
If the velocity-depth profile in a deep ocean has a well defined minimum at finite depth then acoustic signals of high enough frequency are trapped in an associated sound channel and propagate with relatively little attenuation over large distances. An inverse problem of determining the velocity-depth profile given sound channel observations is considered here. This is an inverse eigenvalue problem in which the eigenvalue data (typically recorded as group velocity data) depends on the frequency as an auxiliary parameter, and the inversion
\end{abstract}

* School of Mathematical Sciences, Australian National University, Canberra, ACT 0200, Australia.

${ }^{0}$ See http: //anziamj . austms .org. au/V42/CTAC99/Osbo for this article and ancillary services, (C) Austral. Mathematical Soc. 2000. Published 27 Nov 2000. 
has the possibility of being rescued from the characteristic extreme ill-conditioning of the inverse eigenvalue problem by sampling in the frequency domain. However, the inversion appears to have the unusual characteristic that if a $p$ parameter model is to be determined then it is desirable to have sequences of observations on each of at least $p$ propagating modes.

\section{Contents}

1 Introduction

C1098

2 The inverse problem

C1104

3 Solution of the variational equation

C1111

References

C1113

\section{Introduction}

The Helmholtz equation governing the propagation of sound in a deep ocean in which the velocity of propagation $c(z)$ is a function of the depth $z$ only 
can be separated in cylindrical polar coordinates $(r, z)$ giving the system

$$
\begin{array}{r}
\nabla_{r}^{2} \phi(r)+k^{2} \phi(r)=0, \\
\frac{d^{2} \psi(z)}{d z^{2}}+\left\{\frac{\omega^{2}}{c(z)^{2}}-k^{2}\right\} \psi(z)=0 .
\end{array}
$$

Here $\omega$ is the frequency, the separation constant $k^{2}$ corresponds to wave number, and the pressure release condition at the ocean surface gives a top boundary condition $\psi(0)=0$. The equation governing the radial behaviour can be solved explicitly in terms of Bessel functions and it is the $z$-dependence equation which is of principal interest. Qualitative features of the dependence of $c(z)$ on $z$ are illustrated in Figure 1.

The equation of this curve is

$$
c(z)=4500\left(1+2\left(\eta+e^{-\eta}-1\right)\right)+500 e^{-\frac{4 z}{z_{1}}},
$$

where $\eta=\left(z-z_{1}\right) / z_{B}, z_{1}=1000, z_{B}=12500$. Here $z_{B}$ is the bottom depth, and $z_{1}$ is a good approximation to the sound velocity minimum which determines the axis of the sound channel. Depths are in feet and speeds in feet per second.

If the bottom boundary condition in the deep ocean is approximated by an outgoing wave condition at $z=\infty$ then the Green's function with source 


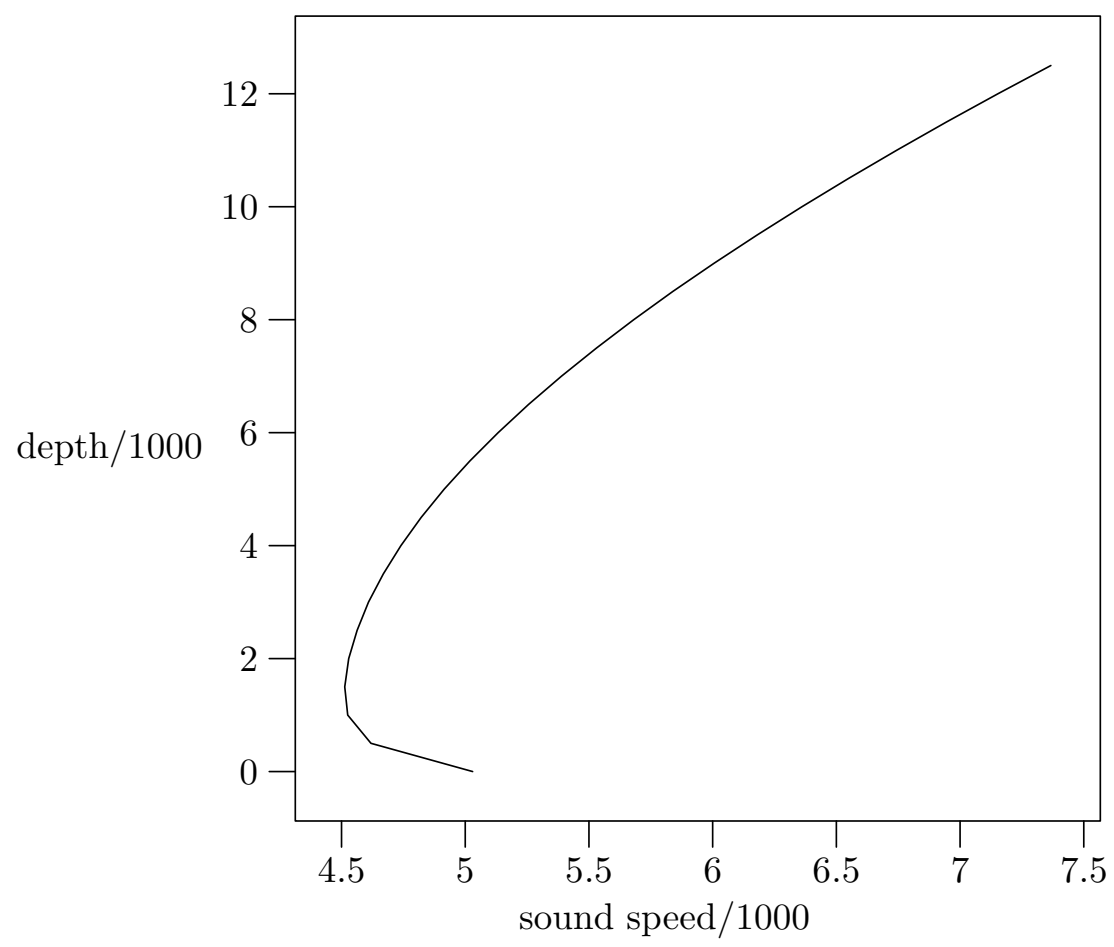

Figure 1: Schematic velocity/depth profile. 
at $\left(0, z_{p}\right)$ has the form

$$
\begin{aligned}
G\left(r, z, z_{p}, \omega\right) & =-\frac{i}{4} \sum_{j} H_{0}^{(2)}\left(k_{j} r\right) \frac{\psi_{j}(z) \psi_{j}\left(z_{p}\right)}{\int_{0}^{\infty} \psi_{j}(z)^{2} d z} \\
& -\frac{i}{4} \int_{0}^{\infty} H_{0}^{(2)}(k r) \psi(z) \psi\left(z_{p}\right) d \sigma(\lambda) .
\end{aligned}
$$

Terms in the sum correspond to the discrete spectrum of the depth dependence equation and are characterised by exponential decay for large $z$. This makes them relatively independent of the precise form of the bottom boundary condition. These modes describe the signals trapped in the sound channel. The weight $\sigma$ associated with the continuous spectrum contribution can be computed if the asymptotic form of $\psi$ for large $z$ is known [2]. The variable of integration is given by $\lambda=\frac{\omega^{2}}{c(\infty)^{2}}-k^{2}$. If the sound velocity is assumed to have a single minimum at $z=z^{*}$ and then to increase monotonically to $z=\infty$ as in Figure 1, then the requirement for an exponential decay means that modes in the discrete spectrum must lie in the range

$$
\frac{\omega^{2}}{c(\infty)^{2}}<k^{2}<\frac{\omega^{2}}{c\left(z^{*}\right)^{2}} .
$$

The characteristic exponential decay of the trapped modes implies cylindrical scattering with a characteristic dispersion of the wavefront and falling off in intensity of $O\left(r^{-1}\right)$. This contrasts with spherical scattering which is nondispersive, and gives an intensity fall-off of $O\left(\left[\sqrt{r^{2}+\left(z-z_{p}\right)^{2}}\right]^{-2}\right) \cdot \mathrm{A}$ 
connection between the continuous spectrum in (3) and an outgoing spherical wave has been shown [3]. Ignoring this term and using the asymptotic form for the Hankel function gives the leading term in the response to the point source for large $r$ as

$$
P \sim \frac{-i}{8 \pi} \sum_{j} \sqrt{\frac{2}{\pi k_{j} r}} \int_{-\infty}^{+\infty} g(\omega) \frac{\psi_{j}(z) \psi_{j}\left(z_{p}\right)}{\int_{0}^{\infty} \psi_{j}(z)^{2} d z} e^{-i\left\{\frac{\pi}{4}+k_{j}-\omega t\right\}} d \omega
$$

where $g(\omega)$ is the Fourier transform of the source. The standard technique for estimating these integrals is the method of stationary phase. This marks as important points satisfying the constructive interference condition

$$
\frac{d}{d \omega}\left\{\omega t-k_{j} r\right\}=0
$$

This leads to the familiar condition for dispersive wave propagation that the component of the disturbance associated with mode $k_{j}$ travels according to the rule

$$
r=\frac{d \omega}{d k_{j}} t=c_{g}^{j} t
$$

where $c_{g}^{j}$ is the group velocity of the $j$ 'th mode. Observation of the group velocities as functions of $\omega$ provides the basic data for the inverse problem. The asymptotic expansion is simplest if $\frac{d c_{g}^{j}}{d \omega} \neq 0$. However, if it vanishes then further constructive interference is possible. Plots of phase and group velocity for the fundamental mode for deep boundary conditions $\psi\left(z_{B}\right)=0$ (dotted 


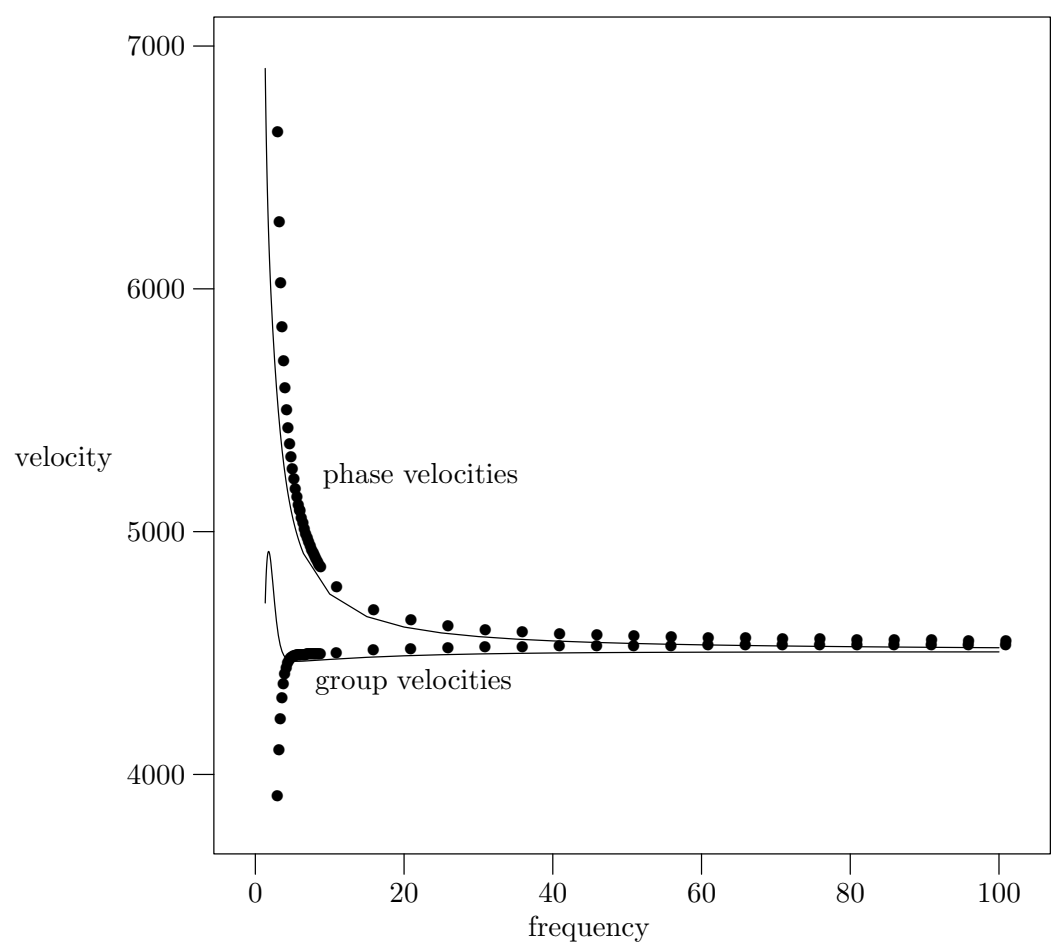

Figure 2: First mode phase and group velocities 
curve) and $\frac{d \psi}{d z}\left(z_{B}\right)=0$ (continuous curve) as a function of frequency are given in Figure 2.

Independence of the bottom boundary condition is apparent for the group velocity only for $\omega>10$. Here the phase velocity is given by $c_{p}^{j}=\omega / k_{j}$, while the group velocity is given by

$$
c_{g}^{j}=\int_{0}^{z_{B}} \psi_{j}(z)^{2} d z /\left\{c_{p}^{j} \int_{0}^{z_{B}} \frac{\psi_{j}(z)^{2}}{c(z)^{2}} d z\right\} .
$$

At higher frequencies the phase and group velocities are close. This follows because the propagating modes are concentrated about the sound channel axis as $\omega$ increases. This is illustrated for the fundamental mode in Figure 3. The frequencies plotted are 5(10)65 cycles per second.

\section{The inverse problem}

There appears to be a distinct similarity between the problem considered here and the classic inverse problem of finding the potential function in a Sturm-Liouville problem given the eigenvalues, but this is misleading:

1. The standard inverse eigenvalue problem is notoriously ill-conditioned so that very accurate data is needed in order to be able to extract 


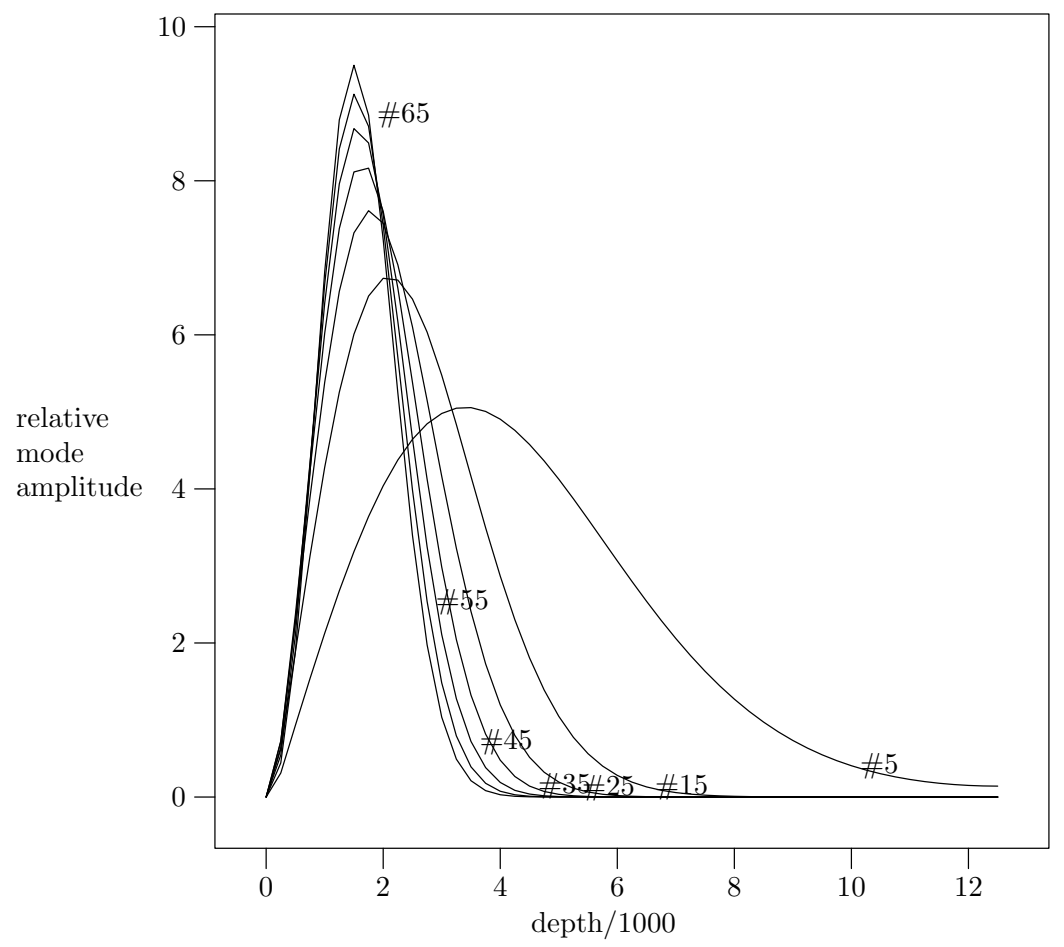

FiguRE 3: Concentration of first mode with increasing frequency. 
any information. The source of the problem is the ill-conditioning of a matrix whose elements are the squares of the eigenvector components.

2. The eigenvalues of (2) give phase velocities. However, it is the group velocities which are the quantities which can be measured. There is no difficulty in principle in using these in the estimation procedure; but computational experience does not indicate any advantage [5].

3. Useful information is available at low frequencies when there are at most a small number of propagating modes.

4. The usual approach does not make use of the frequency dependence.

A key step to improving the inversion could be the use of group velocity data collected as a function of frequency. Let $c\left(\alpha^{*}, z\right)$ be a parametric model for the velocity depth dependence, $\alpha^{*}$ be the true parameter value, and group velocity observations, which depend on $c\left(\alpha^{*}, z\right)$ through the differential equation, be given by

$$
v_{s}^{i}=c_{g}^{s}\left(\alpha^{*}, \omega_{i}^{s}\right)+\varepsilon_{i}^{s}, i=1,2, \cdots, m_{s},
$$

for mode $s, 1 \leq s \leq n_{m}$, for frequency samples

$$
\omega_{\min }^{s} \leq \omega_{1}^{s}<\omega_{2}^{s}<\cdots<\omega_{m_{s}}^{s} \leq \omega_{\max }^{s}
$$

The $\varepsilon_{i}^{s}$ are independent random errors. The approach considered for the inversion is to estimate $\alpha^{*}$ by minimising $F(\alpha): R^{p} \rightarrow R$ with respect to $\alpha$, 
where

$$
F(\alpha)=\sum_{s=1}^{n_{m}} \sum_{i=1}^{m_{s}}\left(v_{i}^{s}-c_{g}^{s}\left(\alpha, \omega_{i}^{s}\right)\right)^{2},
$$

using the Gauss-Newton algorithm. Hypothesise that the number of observations is able to increase without bound and satisfies the conditions:

$$
m=\sum_{s=1}^{n_{m}} m_{s}, \quad \theta_{s}=\lim _{m \rightarrow \infty} \frac{m_{s}}{m}>0 .
$$

For this to be possible there must a defined sampling regime in place for each mode. Here it is assumed that each sampling regime is associated with a limiting weight $\rho_{s}\left(\omega^{s}\right)$. The Gauss-Newton algorithm computes a correction to the parameter estimate $\alpha$ by

$$
\delta \alpha=-\left\{\mathcal{E}\left(\nabla_{\alpha}^{2} F\right)\right\}^{-1} \nabla_{\alpha} F^{T} .
$$

It is required that the limiting Hessian estimate, which is given by

$$
\lim _{m \rightarrow \infty} \frac{1}{m} \mathcal{E}\left(\nabla_{\alpha}^{2} F\right)_{i j}=\sum_{s=1}^{n_{m}} \theta_{s} \int_{R\left(\omega^{s}\right)} \frac{\partial c_{g}^{s}}{\partial \alpha_{j}} \frac{\partial c_{g}^{s}}{\partial \alpha_{k}} d \rho_{s}(\omega), 1 \leq j, k \leq p,
$$

be positive definite, and that it is a good estimate in the sense that the law of large numbers holds in the form

$$
\lim _{m_{s}} \frac{1}{m_{s}} \sum_{l=1}^{m_{s}} \varepsilon_{l}^{s} \frac{\partial^{2} c_{g}^{s}\left(\alpha^{*}, \omega_{i}^{s}\right)}{\partial \alpha_{j} \partial \alpha_{k}} \rightarrow 0, s=1,2, \cdots, n_{m}
$$


In this case it is known that the iteration is asymptotically second order and possesses excellent scaling and stability properties [4].

Some insight into the chance of satisfying these conditions can be obtained by considering the artificial model problem in which

$$
q(z)=\sum_{i=1}^{p} \alpha_{i} q_{i}(z)
$$

is to be estimated given observations $\Lambda_{j}\left(\omega_{i}^{j}\right)+\varepsilon_{i}^{j}, i=1,2, \cdots, m_{j}$, on the system governed by the eigenvalue problem

$$
\frac{d^{2} \psi_{j}}{d z^{2}}+\left(\lambda_{j}-\omega q(z)\right) \psi_{j}=0
$$

subject to boundary conditions $\psi_{j}(0)=\psi_{j}\left(z_{B}\right)=0$. Here $\frac{\partial \lambda_{j}}{\partial \alpha_{i}}(\omega)$ is given by

$$
\frac{\partial \lambda_{j}}{\partial \alpha_{i}}(\omega)=\omega \frac{\int_{0}^{z_{B}} q_{i}(z) \psi_{j}(z, \omega)^{2} d z}{\int_{0}^{z_{B}} \psi_{j}(z, \omega)^{2} d z}
$$

The Gauss-Newton correction solves the linear least squares problem

$$
\min _{\delta \alpha}\|\mathbf{r}\|_{2}^{2} ; \quad \mathbf{r}=\Lambda-\lambda(\omega, \alpha)-\nabla_{\alpha} \lambda(\omega, \alpha) \delta \alpha
$$


The contribution of each mode to the design matrix $\nabla_{\alpha} \lambda(\omega, \alpha)$ involves rows like

$$
\left[\begin{array}{ccc}
\cdots & \cdots & \cdots \\
\frac{\partial \lambda_{j}}{\partial \alpha_{1}}\left(\omega_{s}\right) & \cdots & \frac{\partial \lambda_{j}}{\partial \alpha_{p}}\left(\omega_{s}\right) \\
\cdots & \cdots & \cdots
\end{array}\right]=\left[\begin{array}{ccc}
\cdots & \cdots & \cdots \\
\cdots & \omega_{s} \frac{\int_{0}^{z B} q_{i}(z) \psi_{j}\left(z, \omega_{s}\right)^{2} d z}{\int_{0}^{z} B} \psi_{j}(z, \omega)^{2} d z & \cdots \\
\cdots & \cdots & \cdots
\end{array}\right]
$$

This will contain a rank $(p)$ submatrix provided there exists a set of points $\Omega=\left\{\omega_{1}<\omega_{2}<\cdots<\omega_{p}\right\}$ such that

$$
\max _{\omega_{s} \in \Omega}\left|\int_{0}^{z_{B}} q(z) \psi_{j}\left(z, \omega_{s}\right)^{2} d z\right|>0, \forall q(z) \in \operatorname{span}\left\{q_{i}(z), i=1,2, \cdots, p\right\} .
$$

If $\psi_{j}$ is independent of $\omega$ then this sufficient condition cannot be satisfied for a single mode if $p>1$. Formally the condition can be satisfied if there are observations on at least as many modes as there are parameters in case $p>1$. Note that although the squares of the eigenvectors reappear, it is now the interaction between these and the basis functions $q_{i}(z), i=1,2, \cdots, p$ that is important. In practice $\psi_{j}$ varies slowly with $\omega$ (Figure 3 ) so this argument only holds to some approximation. However, it does suggest that an inversion based on a single mode could be badly conditioned for $p>1$, and that observations on at least $p$ propagating modes would be needed to estimate satisfactorily a $p$ parameter model.

Something similar occurs in the inversion problem which uses the group velocity data. In this case the requirement is for the matrix with elements 
$\frac{\partial c_{g}^{j}}{\partial \alpha_{i}}\left(\omega_{s}\right)$ to have full rank $p$. The group velocity is given by

$$
c_{g}=\frac{1}{c_{p} f(\alpha)}, f(\alpha)=\frac{\int_{0}^{z_{B}} \frac{\psi(z, \alpha)^{2}}{c(z, \alpha)^{2}} d z}{\int_{0}^{z_{B}} \psi(z, \alpha)^{2} d z}
$$

so that

$$
\frac{\partial c_{g}}{\partial \alpha_{i}}=-2 c_{g}\left\{\frac{\frac{\partial c_{p}}{\partial \alpha_{i}}}{c_{p}}+\frac{\frac{\partial f}{\partial \alpha_{i}}}{f}\right\}=c_{g} \int_{0}^{z_{B}} K(z, \alpha) \frac{\partial c}{\partial \alpha_{i}}(z, \alpha) d z
$$

Here the calculation of $K(z, \alpha)$ involves not only $\frac{\partial k}{\partial \alpha}$ but also $\frac{\partial \psi}{\partial \alpha}$ as a consequence of the definition of $f(\alpha)$. The dependence on $\frac{\partial c}{\partial \alpha_{i}}$ follows from the variational equation

$$
\frac{d^{2}}{d z^{2}} \frac{\partial \psi}{\partial \alpha_{i}}+\left(\frac{\omega^{2}}{c(z, \alpha)^{2}}-k^{2}\right) \frac{\partial \psi}{\partial \alpha_{i}}=2\left(\frac{\omega^{2}}{c(z, \alpha)^{3}} \frac{\partial c}{\partial \alpha_{i}}(z, \alpha)+k \frac{\partial k}{\partial \alpha_{i}}\right) \psi
$$

and the condition for solvability

$$
k \frac{\partial k}{\partial \alpha_{i}}=-\frac{\int_{0}^{z_{B}} \frac{\omega^{2}}{c(z, \alpha)^{3}} \frac{\partial c}{\partial \alpha_{i}}(z, \alpha) \psi(z, \alpha)^{2} d z}{\int_{0}^{z_{B}} \psi(z, \alpha)^{2} d z} .
$$

Hence the argument for the idealised problem can be followed through. Here it is necessary to avoid the possibility of a function in the span of the set $\left\{\frac{\partial c_{g}^{j}}{\partial \alpha_{i}}, j=1,2, \cdots, m_{j}\right\}$ being orthogonal to $K^{j}(z, \alpha), j=1,2, \cdots, n_{m}$. 


\section{Solution of the variational equation}

Because $k_{j}^{2}$ is an eigenvalue of (2), the right hand side of (5) must satisfy a compatibility condition (this determines $\frac{\partial k_{j}}{\partial \alpha_{i}}$ ), and the solution is not unique. Thus an additional condition must be added to normalise it, and the obvious condition is

$$
\int_{0}^{z_{B}} \psi \frac{\partial \psi}{\partial \alpha} d z=0
$$

which fits neatly with the scaling condition $\int_{0}^{z_{B}} \psi(z, \alpha)^{2} d z=1$ holding for all $\alpha$. However, the obvious discretisation of this condition leads to an algebraic condition of the form

$$
h \psi^{T} D \frac{\partial \psi}{\partial \alpha}=0
$$

where the vector components are values of the corresponding differential equation quantities, and $D$ is a diagonal matrix summarising the quadrature. This condition makes it hard to preserve sparsity in guaranteed stable methods for solving the discretised extended system. A better plan is to introduce a new variable

$$
w(z)=\int_{0}^{z} \psi(t) \frac{\partial \psi}{\partial \alpha}(t) d t .
$$

This gives us another differential equation

$$
\frac{d w}{d z}-\psi(z) \frac{\partial \psi}{\partial \alpha}(z)=0
$$


and another boundary condition

$$
w(0)=0 .
$$

The equivalent extended system (5) and (7) can be written as a first-order system

$$
\begin{aligned}
\frac{d u}{d z}-v & =0 \\
\frac{d v}{d z}+\left\{\frac{\omega^{2}}{c(z)^{2}}-k^{2}\right\} u & =2\left(\frac{\omega^{2}}{c(z, \alpha)^{3}} \frac{\partial c}{\partial \alpha_{i}}(z, \alpha)+k \frac{\partial k}{\partial \alpha_{i}}\right) \psi \\
\frac{d w}{d z}-\psi(z) u & =0 .
\end{aligned}
$$

The corresponding boundary conditions are

$$
u(0)=u\left(z_{B}\right)=w(0)=0 .
$$

In this form the extended system can be discretised compactly. A discussion of the numerical solution of the eigenvalue problem (5), and the computation of the cut-off frequencies of the propagating modes can be found in the report [1]. 


\section{References}

[1] D. L. Harrar II and M. R. Osborne. Computational techniques for differential equation eigenvalue problems on vector processors. Tech. Report MRR.032.99, School of Mathematical Sciences, Australian National University, 1999. C1112

[2] M. R. Osborne. On the propagation of sound in a layered fluid medium. Q. J. Mech. Appl. Math., 13:472-486, 1960. C1101

[3] M. R. Osborne. On the equivalence of two methods for solving sound propagation problems in a layered fluid medium. Q. J. Mech. Appl. Math., 15:509-518, 1962. C1102

[4] M. R. Osborne. Fisher's method of scoring. Int. Stat. Rev., 60:99-117, 1992. C1108

[5] M. R. Osborne and A. Tan. An inverse problem associated with sound channel propagation. In D. Stewart, H. Gardner, and D. Singleton, editors, Computational Techniques and Applications: CTAC93, pages 397-405. World Scientific, Singapore, 1994. C1106 